\title{
Rapid Diagnosis of Bacterial Pneumonia in Under-five Children by Latex Particle Agglutination Test in Urine
}

\author{
Shahin Ara Begum, Aliya Rashid, Md. Abdur Rhman, Akhtaruzzman Chowdhury, Sufi HZ Rahman, Naima Muazzam \\ Department of Microbiology, Dhaka Medical College, Dhaka
}

\begin{abstract}
Streptococcus pneumoniae and Haemophilus influenzae type b are the main causes of community-acquired bacterial pneumonia in developing countries including Bangladesh. This study was carried out to find a rapid diagnostic method for early detection of bacterial pneumonia. Latex Particle Agglutination Test (LPAT) was done in urine for quick detection of capsular antigens of $S$. pneumoniae and $\boldsymbol{H}$. influenzae type $\mathrm{b}$. Three hundred children were enrolled based on clinical criteria as defined by the WHO. Among them, 70 pneumonic children, who had total white blood cell counts around $20000 / \mathrm{mm}^{3}$, Neutrophils $\geq 75 \%$ and consolidation on chest X-rays, were selected for LPAT in urine. Some 20 children of the same age group, who had chest diseases other than pneumonia, were selected as sick control group. Among 70 pneumonic cases, 36 (51.43\%) were positive by LPAT in urine. Out of 36 LPAT-positive cases, 19 (52.7\%) were positive for $S$. pneumoniae and 17 (47.3\%) were positive for $H$. influenzae type b by specific anti-sera. The LPAT is thus appears as a quick and reliable test for the diagnosis of bacterial pneumonia cases caused by $S$. pneumoniae and $\mathrm{H}$. influenzae type $\mathrm{b}$.
\end{abstract}

Key words: Emerging resistance, Common bacteria

\section{Introduction}

Pneumonia is one of the leading causes of morbidity and mortality in children worldwide, mainly in developing countries. ${ }^{1,2}$ In 1990, 12.9 million children under the age of five died, a third of this deaths were due to acute respiratory tract infection (ARI), mainly pneumonia. ${ }^{3}$ It is estimated that at least three million children die from pneumonia each year in developing countries. ${ }^{4}$ Mortality from pneumonia is approximately 10-15 times higher in developing countries than in developed countries.5,6 In Bangladesh, acute respiratory tract infection (ARTI) is one of the most frequent cause of death. ${ }^{7}$ The Community Acquired Pneumonia (CAP) presents both a diagnostic and therapeutic challenge to clinicians. ${ }^{8}$ Determination of the etiology of lower respiratory tract infection is a complex task. ${ }^{9}$ Definite clinical diagnosis

Correspondence:

Dr. Shahin Ara Begum, Medical Officer, Department of Microbiology, Dhaka Medical College, Dhaka- 1000

Tel: 02-9665518 (Office), 01911393115 (Mobile) is based on X-Ray findings, culture of lung aspirates and measurement of blood oxygen level. ${ }^{10}$ The chest radiograph considered the "gold standard" for the diagnosis of pneumonia but cannot differentiate the bacterial and nonbacterial origin. ${ }^{11}$ In recent years, blood cultures have been utilized to obtain information on the bacterial etiology of pneumonia in young children but its sensitivity is low, because less than $8 \%$ of the children with Acute lower respiratory tract infection (ALRI) are bacteremic. ${ }^{12,13}$ The ALRI is one of the major causes of morbidity and mortality of the children under five years in the United States and throughout the world. ${ }^{14}$ S. pneumoniae and H. influenzae type $b$ are main the agents among community-acquired pneumonia in developing countries. ${ }^{15}$

Organisms responsible for pneumonia occasionally cause bacteremia. Lung aspiration culture is the most sensitive and reliable method for isolation of bacterial agents in children but lung aspiration is invasive and exposes the child to potentially dangerous complications. ${ }^{8,16,17}$ Several studies on blood culture among children with pneumonia showed that 
positive culture ranged between $1 \%$ to $27 \%{ }^{18}$ Etiological diagnosis of bacterial pneumonia is difficult in small children, as collection of blood for culture is difficult or already started antibiotics, as is widely practiced in Bangladesh. Thus the true burden of the disease is still unknown in many developing countries.19, 20 In this condition, detection of antigen in urine samples is a simple useful tool for diagnosis of bacterial pneumonia. ${ }^{21}$ One study in India revealed that blood culture was positive in only $10(9.09 \%)$ of 110 children who had features of ALRI, whereas by latex agglutinations assay on serum and urine, $52(47.2 \%)$ cases were found positive. ${ }^{22}$ The present study was undertaken to detect antigen in urine for rapid diagnosis of bacterial pneumonia among children of under-five years.

\section{Methods}

The study was carried out in the department of Microbiology, Dhaka Medical College during the period of November, 2004 to November, 2005. It was a cross-sectional study. A total of 300 children of under-five years, who were suffering from pneumonia clinically, were included in this study. The patients were selected from the pediatrics ward of Dhaka Medical College Hospital and Azimpur Matrnity Hospital. A detailed medical history, socio-economic status of parents was taken and physical examination was done on each patient. All data were recorded in a pretested data sheet. According to WHO guidelines, patients were classified into three groups: a) pneumonia; b) severe pneumonia; and c) very severe pneumonia.

Among the 300 pneumonic children, 70, who had total WBC counts around 20,000, neutrophil counts $\geq 75 \%$ and consolidation on chest X-rays, were selected for LPAT in urine.

Twenty children of the same age group with similar socioeconomic background, suffering from heart/chest disease, other than pneumonia, were selected as sick control. Symptoms like cough, temperature, respiration rate, chest indrawing, stridor, wheezing and creeping, cyanosis, unable to drink, were considered to diagnose the cases of pneumonia. ${ }^{21,22}$ Patients suffering from renal failure, foreign body aspiration, respiratory paralysis, chromosomal anomalies were excluded from this study.

Urine was collected aseptically, for antigen detection by latex particle agglutination test (LPAT). Urine samples were kept at $-20^{\circ} \mathrm{c}$ if the test was not done immediately. The Wellcogen bacterial antigen kit (Bronidox) was used to detect antigen of
Haemophilus influenzae type $\mathrm{b}$ and Streptococcus pneumoniae in urine.

Urine were heated at $100^{\circ} \mathrm{C}$ in a boiling water bath for 5 minutes and allowed to cool at room temperature before use. The samples were then centrifuged at $1400 \mathrm{rpm}$ for 15 minutes, and the supernatant fluid was then tested. Positive test showed agglutination and negative test showed no agglutination. Positive control and negative control tests were done by the reagents included in the kits.

\section{Results}

Among 300 cases, 186 (62\%) children were male and 114 (38\%) were female, of them majority (63\%) were between 3 11 months, $28 \%$ were within $<12-23$ months. Majority $(65 \%)$ of the cases came from lower income group, whereas $35 \%$ cases were from middle-income group. There was none from higher income group. Among the cases, 70 (23.3\%) children had both lung consolidation and high leukocyte count. Their WBC were around 20,000 and neutrophil count $\geq 75 \%$. Some $38(12.82 \%)$ cases had high leukocyte count with patchy opacity. The LPAT in urine was done with 70 (23.3\%) cases, who had consolidation on X-ray chest and total WBC $\pm 20,000$ per $\mathrm{mm}^{3}$ of blood and neutrophils $\geq 75 \%$. (Table I)

Table I: X-ray findings, TLC* and neutrophil percentage among 300 clinically diagnosed pneumonia cases

\begin{tabular}{lllc}
\hline No. of study subject & X-Ray findings & WBC $/ \mathrm{mm}^{3}$ & Neutrophil \\
\hline $70(23.3 \%)$ & Consolidation & $\geq 20,000$ & $\geq 75 \%$ \\
$38(12.6 \%)$ & Patchy opacity & 15,000 & $\geq 70 \%$ \\
$192(64.0 \%)$ & Patchy opacity & $\leq 15,000$ & $\leq 70 \%$ \\
\hline & & *TLC- Total leukocyte count
\end{tabular}

Out of 70 cases, LPAT in urine was positive in $36(51.9 \%)$ cases who had high leukocyte count and lung consolidation. Whereas, among 20 cases of control group, only 1 (5\%) was positive by LPAT. (Table II)

Table II: Latex Particle Agglutination Test (LPAT) in urine samples of children with pneumonia $(n=70)$

\begin{tabular}{lccc}
\hline \multirow{2}{*}{ Study Subjects } & Positive & Nogative & Total \\
\hline Cases & $36(51.9 \%)$ & $34(48.1 \%)$ & $70(100.0 \%)$ \\
Control & $01(5.0 \%)$ & $19(95.0 \%)$ & $20(100.0 \%)$ \\
\hline
\end{tabular}


Out of 36 LPAT positive cases, 19 (52.7\%) were $S$. pneumoniae and $17(47.3 \%)$ were $H$. influenzae type b. (Figure 1)
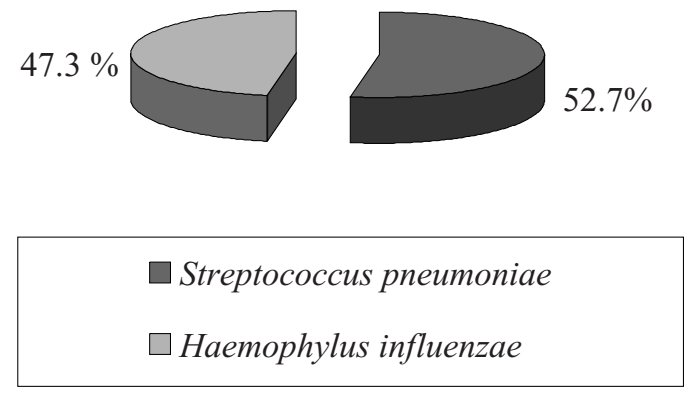

Figure 1: Bacterial agents detected by LPAT

\section{Discussion}

Various community- and hospital-based studies in Bangladesh noted that mortality from pneumonia among under-five children was $10 \%$ to $30 \% .22$ A positive blood culture provides the basis for a specific etiologic diagnosis but it is time-consuming and the sensitivity of this method is low. The method of direct examination and culture of sputum have lower sensitivity and specificity. Antigen detection is a promising noninvasive approach for diagnosis of pneumonia. Bacterial antigens produced at site of infection are distributed to body fluids and can be detected by various test procedures like Latex particle agglutination, Co-agglutination, ELISA and PCR. ${ }^{14}$ In this study, urine was tested for detection of antigens of $H$. influenzae type $\mathrm{b}$ and $S$. pneumoniae by LPAT.

Among 70 cases selected from 300 clinically diagnosed pneumonic children, LPAT in urine were positive in 36 $(51.9 \%)$ and rest $34(34.1 \%)$ were negative. Similarly, Nunes et al (2004) reported that among 45 children with pneumonia, who had positive X-ray findings and high leukocyte count, LPAT were positive in $77.3 \%$ cases and negative in $22.7 \%{ }^{15}$ Another study in India by Bahl et al (1995) showed the highest diagnostic yield with LPAT showing 52 (41.2\%) cases positive by LPAT. ${ }^{23}$ One study in USA found $24 \%$ antigenuria, in children with acute lower respiratory tract infection. ${ }^{12}$ In our study, among 70 pneumonic children, 19 (52.7\%) S. pneumniae and $17(47.3 \%) H$. influenzae type b were detected by LPAT.

The LPAT is a quick and reliable test for the diagnosis of bacterial pneumonia caused by $S$. pneumoniae and $H$. influenzae type $\mathrm{b}$, even in partially-treated patients where cultures are often negative. It is a simple, rapid test and can be carried out in an ordinary laboratory setup. Detection of bacterial pneumonia cases by LPAT in urine may be higher if the kit would provide reagent for other capsulated $H$. influenzae, Staphylococcus aureus, Klebsiella pneumoniae.

\section{References}

1. Shapiro ED. Epidemiology of acute respiratory infections. Semin Pediatr Infect Dis 1998; 9 : 31-36.

2. Shohet FA, Leiberman JM. Bacterial Pneumonia in children. Semin Pediatr Infect Dis 1998; 9: 191-198.

3. Kumar V. A strategy for ensuring promt recognition and treatment of pneumonia. ARI News 1995; 31: 2-3.

4. Greenwood B. The epidemiology of pneumococcal infection in children in developing world. Phil Trans R Soc Land 1999; 354: $777-785$.

5. Pio A. The problem of acute respiratory infection in children in developing countries. Proceedings of an international workshop in Sydney, Australia; 1984: pp. 3-6.

6. WHO. A program for controlling acute respiratory infection in children. Memorandum from a WHO meeting. WHO Bull 1984; 62: 47-58.

7. Huq F, Rahman M, Nahar N, et al. Acute lower respiratory tract infection due to virus among hospitalized children in Dhaka, Bangladesh. Rev Infect Dis 1990; 88 (12): 982-986.

8. Luna RH, Pankey G. The utility of Blood Culture in patients with community acquired pneumonia. Ochsner J 2002; 3: 8593.

9. James RH, Dinash PS, Mehroor C, Keertiv S, Sisirk B. Lung tap in childhood Bacteria, Virus and Mycoplasma in acute lower respiratory tract infections. Pediatrics 1969; 44: 477-485.

10. Vijay K. A strategy for ensuring promt recognition and treatment of pneumonia. ARI News 1995; 31: 2-3.

11. Marrie TJ. Community- Acquired Pneumonia. Clin Infect Dis 1994; 18: 501-515.

12. Ramsey BW, Mercuse MDK, Foy HM, et al. Use of Bacterial antigen detection in the diagnosis of pediatric lower respiratory tract infections. Padiatrics 1986; 78: 1-9.

13. Wall RA, Corrah PT, Mabey DCW, Grenwood BM. The etiology of lobar pneumonia in the Gambia. Bull WHO 1986; 64: 553558.

14. Gloria WA, Gail AB, Hayes PS, et al. Commercial Latex Agglutination test for Detection of Haemophilus influenzae type b and Streptococcus pneumoniae antigen in patients with Bacteremic Pneumonia. J Clin Microbiol 1987; 25: 1388-1391. 
Rapid Diagnosis of Bacterial Pneumonia in Under-five Children

15. Nunes AA, Camargos PAM, Costa PR, Campos MTK. Antigen Detection for Diagnosis of pneumonia. Ped Pul Dis 2004; 38 : $1-5$.

16. Sutmeller F, Ferro ZPA, Asensi MD, Ferreira V, Mazzei IS, Cunha BL. Etiology of acute respiratory tract infections among children in a combined community and hospital study in Rio de Janerio. Clin Infect Dis 1995; 20: 854-860.

17. Ocampo Santos PD. Management of acute respiratory tract infections in primary health-care. Inter Child Health 1992; 8: $52-62$.

18. Carvallo-Nascimento, Cristiana MC. Etiology of Childhood Community Acquired Pneumonia and its Implications for Vaccination. Brazillian J Infect Dis 2001; 5: 1-14.

19. Rajalakshmi B, Kanungo, Srinivara R, Badrinrth S. Pneumolysin in Urine- A Rapid Antigen Detection Method to Diagnose Pneumococcal Pneumonia in Children. Indian J Med Microbiol 2002; 20 (4): 183-186.

20. Greenwood BM. Diagnosis of bacterial pneumonia by Antigen
Begum et al

detection. ARI News 1988; 10: 7.

21. Jose D, Nuria G, Silvia B, Pablo P, Cristina P. Detection of Streptococcus pneumoniae Antigen by a Rapid Immunochromatographic Assay in urine samples. Chest 2001; 119: 243-249.

22. Baqui AH, Black RE, Arifeen SE, Hill, Mitra SN, Sabir A. Causes of childhood deaths in Bangladesh: result of a nationwide verbal autopsy study. WHO Bull 1998; 76: 161-171.

23. Bahl AH, Mishra S, Sharma D, Singhal A, Kumari S. A bacteriological study in hospitalized children with pneumonia. Ann Trop Paediatr 1995; 15 (2): 173-177.

24. Selwyn BJ. The epidemiology of acute respiratory tract infection in young children. Comparison of finding from several developing countries. Rev Infect Dis 1990; 12 (8): 870-890.

25. Anonymous. A program for controlling acute respiratory infection in children. Memorandum from a WHO meeting. Bull WHO 1984; 62: 47-58. 